\title{
OPEN Hepatitis C prevalence and quality of health services among HIV-positive mothers in the Democratic Republic of the Congo
}

Peyton Thompson ${ }^{1 凶}$, Christian Mpody ${ }^{2}$, Wesley Sayre ${ }^{3}$, Clare Rigney ${ }^{4}$, Martine Tabala ${ }^{5}$, Noro Lantoniaina Rosa Ravelomanana ${ }^{5}$, Fathy Malongo ${ }^{5}$, Bienvenu Kawende ${ }^{5}$, Frieda Behets ${ }^{6}$, Emile Okitolonda ${ }^{5}$, Marcel Yotebieng ${ }^{7} \&$ CQI-PMTCT study team ${ }^{5 *}$

Hepatitis $\mathrm{C}$ virus (HCV) contributes to liver-related morbidity and mortality throughout Africa despite effective antivirals. HCV is endemic in the Democratic Republic of the Congo (DRC) but data on HCVI $\mathrm{HIV}$ co-infection in pregnancy is limited. We estimated the prevalence of and risk factors for HCV/ HIV co-infection among pregnant women in the Kinshasa province of the DRC. This cross-sectional study was conducted as a sub-study of an ongoing randomized trial to assess continuous quality improvement interventions (COI) for prevention of mother-to-child transmission (PMTCT) of HIV (COIPMTCT study, NCT03048669). HIV-infected women in the COI-PMTCT cohort were tested for HCV, and risk factors were evaluated using logistic regression. The prevalence of $\mathrm{HCV} / \mathrm{HIV}$ co-infection among Congolese women was $0.83 \%(95 \% \mathrm{Cl}$ 0.43-1.23). Women who tested positive for $\mathrm{HCV}$ were younger, more likely to live in urban areas, and more likely to test positive during pregnancy versus postpartum. HCV-positive women had significantly higher odds of infection with hepatitis B virus (HBV) (aOR $13.87[3.29,58.6])$. An inverse relationship was noted between $\mathrm{HCV}$ infection and the overall capacity of the health facility as measured by the service readiness index (SRI) (aOR:0.92 $[0.86,0.98]$ per unit increase). Women who presented to rural, for-profit and PEPFAR-funded health facilities were more likely to test positive for HCV. In summary, this study identified that the prevalence of HCV/HIV co-infection was $<1 \%$ among Congolese women. We also identified HBV infection as a major risk factor for HCV/HIV co-infection. Individuals with triple infection should be linked to care and the facilityrelated differences in HCV prevalence should be addressed in future studies.

Hepatitis C virus (HCV) accounts for a large proportion of morbidity and mortality from liver disease globally, particularly in sub-Saharan Africa (SSA). Recent data suggests that prevalence of HCV in SSA ranges from $0.72 \%$ in Southern Africa to $7.82 \%$ in Central Africa ${ }^{1}$. A recent national survey in the Democratic Republic of the Congo (DRC) found an HCV prevalence of $0.9 \%$, although prevalence estimates varied by province ${ }^{2}$. Effective antiviral treatment for HCV exists but is not accessible for most inhabitants of SSA, thus perpetuating the epidemic.

Health outcomes in individuals with HCV are far poorer when in the setting of co-infection with HIV. HCV/ HIV co-infection has been associated with accelerated liver fibrosis ${ }^{3}$. Liver-related complications have been demonstrated to be a frequent cause of mortality in co-infected individuals as well ${ }^{4}$. Infection with both HCV and HIV has been seen throughout SSA. Several sub-Saharan countries, including Togo, Nigeria, Cameroon,

${ }^{1}$ Division of Infectious Diseases, Department of Pediatrics, University of North Carolina, Chapel Hill, NC, USA. ${ }^{2}$ Division of Epidemiology, College of Public Health, The Ohio State University, Columbus, OH, USA. ${ }^{3}$ School of Medicine, University of North Carolina, Chapel Hill, NC, USA. ${ }^{4}$ College of Health Sciences, Cleveland State University, Cleveland, OH, USA. ${ }^{5}$ School of Public Health, University of Kinshasa, Kinshasa, Democratic Republic of Congo. ${ }^{6}$ Department of Epidemiology, Gillings School of Global Public Health, University of North Carolina at Chapel Hill, Chapel Hill, NC, USA. ${ }^{7}$ Division of General Internal Medicine, Department of Medicine, Albert Einstein College of Medicine, Bronx, NY, USA. *A list of authors and their affiliations appears at the end of the paper. ${ }^{\circledR}$ email: peyton_thompson@med.unc.edu 
Gabon, Mozambique and the DRC, are considered to be high-prevalence areas ( $>3.5 \%$ according to published definitions) for HCV/HIV co-infection ${ }^{5,6}$.

Although HCV/HIV co-infection has a clear impact on individuals living in SSA, data on the prevalence of and risk factors for HCV/HIV co-infection in pregnancy are limited. Mother-to-child transmission (MTCT) of $\mathrm{HCV}$ is higher among HCV/HIV co-infected mothers compared to HCV mono-infected mothers (10-20\% vs. $5-7 \%$, respectively $)^{7}$. It is important to identify HCV/HIV co-infected pregnant women and to determine risk factors for co-infection in order to mitigate this risk and to prevent future infections. We previously conducted a cross-sectional study investigating hepatitis B virus (HBV) and HIV co-infection in pregnant and postpartum women, demonstrating a high prevalence of co-infection and raising concern for increased risk of vertical transmission of both $\mathrm{HIV}$ and $\mathrm{HBV}^{8}$. Considering the limited data on $\mathrm{HCV} / \mathrm{HIV}$ co-infection, we expanded upon this previous study to determine the prevalence of and risk factors for HCV-HIV co-infection in Congolese women within the Kinshasa province. We also investigated facility-level factors that were associated with HCV status, such as location and type of facility the women attended, as well as the service readiness index, a measure of the quality of care a facility provides.

\section{Methods}

Study design and setting. This cross sectional study was a sub-study of an ongoing randomized trial to investigate the effect of data-driven continuous quality interventions (CQI) on long-term anti-retroviral therapies (ART) outcomes among pregnant and breastfeeding women receiving care in Kinshasa province through the Continuous Quality Improvement-Prevention of Mother to Child Transmission (CQI-PMTCT) study, NCT03048669 ${ }^{9}$. This study gathered data from 105 sites, all of which were located within the Kinshasa province (Supplementary Fig. S1).

The parent CQI-PMTCT study is prospective in nature, enrolling women at any point from early pregnancy to 12 months postpartum. At enrollment, the HCV status of the eligible participants was assessed. The study was approved by the Ohio State University Institutional Review Board and the Kinshasa School of Public Health Ethical Committee. All methods were carried out in accordance with relevant guidelines and regulations. Pregnant women under the age of 18 were considered emancipated minors in the DRC and were consented directly per the 1987 Law of Emancipation (See Supplementary Information). Written informed consent was obtained from all subjects. This method of consent was approved by the Ethics Committee that reviewed the application. The age of emancipation in the DRC changed to $\geq 18$ years in 2019, but enrollment and activities for this study had been completed by that time.

Inclusion and exclusion criteria. All HIV-infected pregnant or breastfeeding women receiving care at any of the selected MCH facilities between November 2016 and July 2019 were eligible for the parent study, as described previously. Women were excluded if they refused to participate or if they were beyond 12 months postpartum. For this study, women were also excluded if they refused to be tested for HCV.

Data collection. Eligible participants were referred to study staff for informed consent and enrollment in the study upon presentation for routine visits any time during pregnancy and 12 months postpartum. A participant questionnaire for baseline demographic and clinical data was administered to individuals who consented to be involved in the study. The participants completed survey questions via paper questionnaires administered by the study personnel. These questionnaires were comprised of mostly structured questions, with a few openended components. An adapted version of the World 'Health Organization (WHO)'s Service Availability and Readiness Assessment (SARA) questionnaire was used to assess the capacity of health facilities to provide general health services ${ }^{10}$.

Capacity of health facilities to provide general maternal and child health services. Between August and November 2016, we surveyed the 105 sites of enrollment using an adapted version of SARA. Our adapted instrument contained 185 items organized into 12 domains: (1) General information and service availability of the health facility, (2) General service readiness, (3) Staffing, management and quality assurance, (4) Diagnostic services, (5) Antenatal care, (6) PMTCT of HIV infection, (7) Delivery and newborn care services, (8) Well baby clinic, (9) Child curative care services, (10) Family planning services (11), and (12) HIV treatment, care and support.

The service readiness index (SRI) was modeled after the WHO's service readiness availability index [25], which contains 50 items that are grouped into five categories: (1) basic amenities, (2) basic equipment, (3) infection prevention, (4) diagnostic capacity, and (5) essential medicines (Supplementary Table S1). We identified and matched 35 questions that covered the first four domains-i.e. leaving out the domain of essential medicines. Replicating the WHO's method, we first computed a percentage score for each domain that is based on the availability of items within a domain. Then, we averaged percentage scores across the four domains to obtain the composite SRI. The Combrach's alpha ( $\alpha=0.77,95 \%$ CI $0.71,0.83)$ indicated acceptable internal consistency.

Outcome variables. The primary outcome of interest for this study was the HCV antibody status of participants (positive or negative). This was measured using the SD Bioline HCV rapid antibody test, verified for use within the region ${ }^{11,12}$.

Covariates. Other variables considered in this analysis, as in a previous analysis of HBV/HIV co-infection, included participants' socio-demographic information, clinical features and health facility characteristics. Participant socio-demographic characteristics included the following: maternal age $(\leq 24,25-34, \geq 35)$, marital sta- 


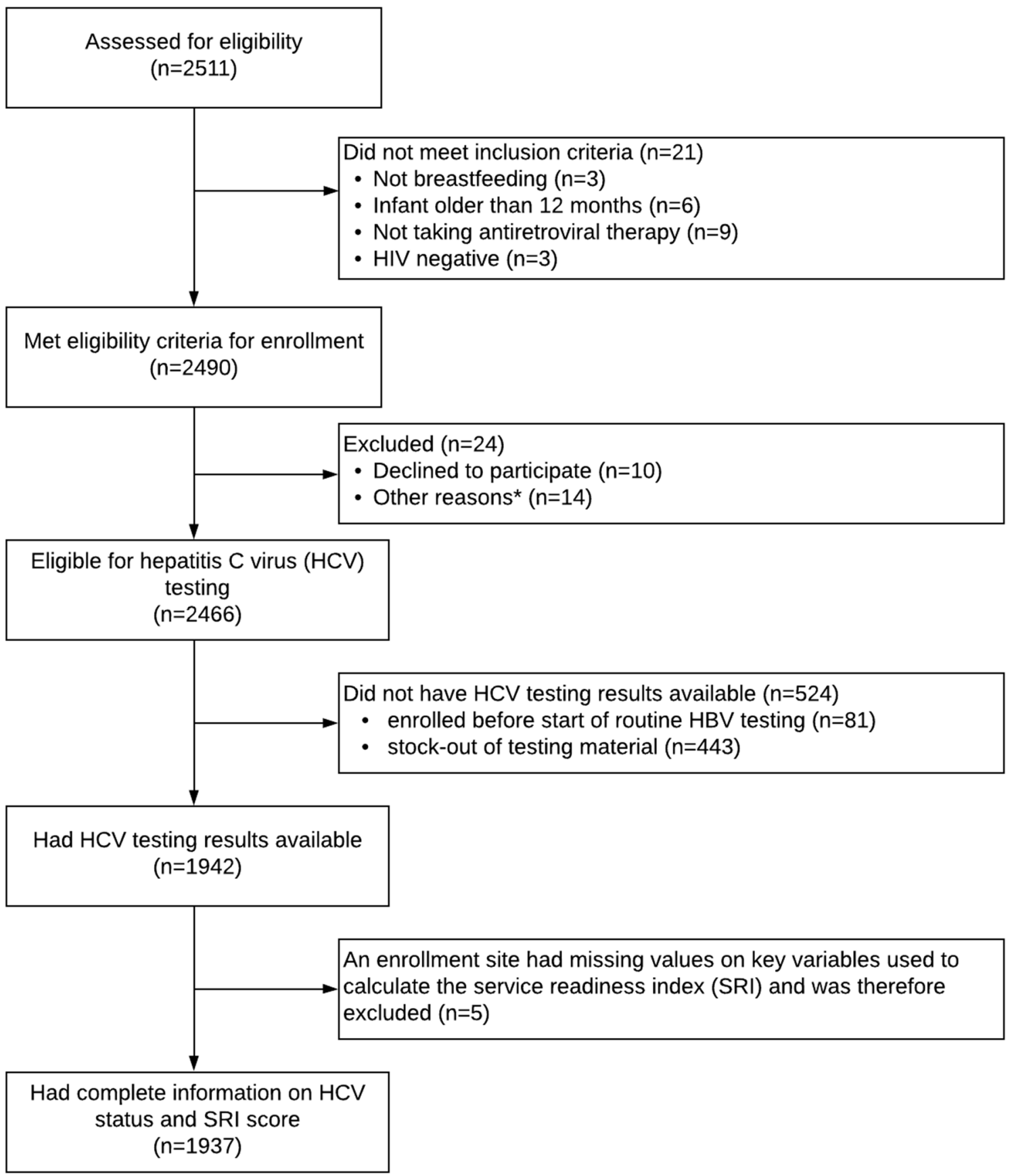

Figure 1. Flow chart of eligibility and enrollment.

tus (married/cohabitating vs. divorced/separated/widowed/never married), educational level (primary, secondary, or tertiary), alcohol consumption (no vs. yes), and socioeconomic status (SES) - measured by a wealth index score. Calculation of the wealth index score is described by Mpody et al. ${ }^{13}$.

Clinical characteristics included: HBV infection (yes vs. no - using the Alere HBsAg rapid test) ${ }^{14}$, duration of ART in months ( $\leq 6,6-24$, or $>24$ ), disclosure of HIV status to anyone (yes vs. no), primigravida (yes vs. no), report of any intimate partner violence (IPV; yes vs. no) and HIV virological suppression [Viral load (VL) $\leq 1000$ copies/mL (suppressed) vs. VL $>1000$ copies/mL (unsuppressed)].

Facility factors included location (urban vs. peri-urban/rural), type of facility (hospital vs. health center, as per self-report by the facility manager), and whether they receive direct PEPFAR funding support for HIV care (yes vs. no). Of note, maternity clinics in Kinshasa receive funding for the provision of HIV care either from PEPFAR or from the Global Fund. Non-HIV care is provided via fee-for-service care across the various types of facilities.

Statistical analysis. Statistical analyses were similar to those of a previous study of HBV/HIV co-infection in the same population ${ }^{8}$. We calculated the proportion of participants with a positive HCV antibody test as a measure of HCV prevalence with associated $95 \%$ binomial confidence intervals (CIs). Bivariate and multivari- 


\begin{tabular}{|c|c|}
\hline \multicolumn{2}{|l|}{ Study participant characteristics } \\
\hline \multicolumn{2}{|l|}{ Age } \\
\hline $35+$ & $305(16.1)$ \\
\hline $25-34$ & $997(52.7)$ \\
\hline$\leq 24$ & $589(31.1)$ \\
\hline Missing & 51 \\
\hline \multicolumn{2}{|l|}{ Timing of testing } \\
\hline Pregnancy & $1154(59.4)$ \\
\hline Delivery & $417(21.5)$ \\
\hline Post-partum & $371(19.1)$ \\
\hline Missing & 0 \\
\hline \multicolumn{2}{|l|}{ Marital status } \\
\hline Married/cohabitating & $608(32.2)$ \\
\hline Divorced/separated/widow/never married & $1283(67.8)$ \\
\hline Missing & 51 \\
\hline \multicolumn{2}{|l|}{ Alcohol consumption } \\
\hline No & $1369(72.4)$ \\
\hline $1-3 /$ month & $416(22)$ \\
\hline$>2 /$ week & $106(5.6)$ \\
\hline Missing & 51 \\
\hline \multicolumn{2}{|l|}{ Smoking status } \\
\hline Previous/current & $36(1.9)$ \\
\hline Never & $1846(98.1)$ \\
\hline Missing & 60 \\
\hline \multicolumn{2}{|l|}{ Educational level } \\
\hline Tertiary & $245(13)$ \\
\hline Secondary & $1348(71.4)$ \\
\hline Primary & $296(15.7)$ \\
\hline Missing & 53 \\
\hline \multicolumn{2}{|l|}{ SES in tertiles } \\
\hline 3 (Highest) & $607(33.4)$ \\
\hline 2 & $608(33.5)$ \\
\hline 1(Lowest) & $600(33.1)$ \\
\hline Missing & 127 \\
\hline \multicolumn{2}{|l|}{ Primigravida } \\
\hline Yes & $195(10.3)$ \\
\hline No & $1700(89.7)$ \\
\hline Missing & 47 \\
\hline \multicolumn{2}{|l|}{ Any intimate partner violence ${ }^{\#}$} \\
\hline No & $1196(63.2)$ \\
\hline Yes & $696(36.8)$ \\
\hline Missing & 50 \\
\hline \multicolumn{2}{|l|}{ HIV RNA viral load } \\
\hline$\geq 1000$ copies $/ \mathrm{mL}$ & $678(35.9)$ \\
\hline$<1000$ copies $/ \mathrm{mL}$ & $1208(64.1)$ \\
\hline Missing & 56 \\
\hline \multicolumn{2}{|l|}{ ART regimen } \\
\hline $\mathrm{TDF}+3 \mathrm{TC}+\mathrm{FEV}$ & $1601(82.4)$ \\
\hline $\mathrm{AZT}+3 \mathrm{TC}+\mathrm{NVP}$ & $201(10.4)$ \\
\hline Other & $140(7.2)$ \\
\hline \multicolumn{2}{|l|}{ Duration of ART } \\
\hline$<12$ months & $846(45)$ \\
\hline 13-24 months & $215(11.4)$ \\
\hline$\geq 24$ months & $819(43.6)$ \\
\hline Missing & 62 \\
\hline \multicolumn{2}{|l|}{ Disclosure of HIV status ${ }^{* *}$} \\
\hline Continued & \\
\hline
\end{tabular}




\begin{tabular}{|c|c|}
\hline & $\begin{array}{l}\text { No(\%) } \\
n=1942\end{array}$ \\
\hline No & $910(47)$ \\
\hline Yes & $1028(53)$ \\
\hline Missing & 4 \\
\hline \multicolumn{2}{|l|}{ HBV status } \\
\hline Negative & $1858(95.8)$ \\
\hline Positive & $81(4.2)$ \\
\hline \multirow[t]{2}{*}{ Missing } & 3 \\
\hline & $\begin{array}{l}\text { No (\%) } \\
n=1942\end{array}$ \\
\hline \multicolumn{2}{|c|}{$\underline{\text { Facility characteristics }}^{\S}$} \\
\hline \multicolumn{2}{|c|}{ Location of facility attended } \\
\hline Peri-urban/rural & $135(7)$ \\
\hline Urban & $1802(93)$ \\
\hline Missing & 5 \\
\hline \multicolumn{2}{|c|}{ Type of facility of care } \\
\hline Health center & $821(42.4)$ \\
\hline Hospital & $1116(57.6)$ \\
\hline Missing & 5 \\
\hline \multicolumn{2}{|l|}{ PEPFAR funding } \\
\hline No & $731(37.7)$ \\
\hline Yes & $1206(62.3)$ \\
\hline \multicolumn{2}{|c|}{ Type of facility of care } \\
\hline Public & $623(32.2)$ \\
\hline Private for profit & $249(12.9)$ \\
\hline Private non-profit & $1065(55)$ \\
\hline
\end{tabular}

Table 1. Sociodemographic, clinical and facility characteristics of the study population. The analytical sample was derived from the enrollment data of an ongoing cluster randomized controlled trial, aimed at evaluating the effect of data-driven continuous quality improvement on long-term ART outcomes in Kinshasa, Democratic Republic of Congo. We retained participants that had available data on HCV rapid testing. SES, socio-economic status; RNA, ribonucleic acid, ART, antiretroviral therapy, HBV, hepatitis B virus. ${ }^{*}$ Selfreport of disclosure of HIV status to anyone. ${ }^{\S} \mathrm{Facility}$ at which participant was enrolled/tested. ${ }^{\circ} \mathrm{Cal}$ culated using principal component analysis and categorized in three groups: the lower first two quintiles, the middle quintiles, and the last two quintiles. ${ }^{\#}$ Self-report of emotional or physical or sexual partner violence.

able logistic regression modeling was used to estimate prevalence odd ratios (OR) and Wald's 95\% CI comparing the prevalence of HCV antibody across levels of covariates. Covariates were included in the multivariable model if the p-value was $<0.20$ in bivariate analysis. Generalized estimating equation was used to account for potential clustering at the level of health facilities. Only participants with complete data on all covariates were included in the final analysis. All analyses were conducted using SAS, version 9.3 (SAS Institute Inc., Cary, North Carolina), and STATA/IC version 14.0 (StataCorp LP, College Station, Texas). All tests, unless otherwise indicated, were conducted using a two-sided 0.05 significance level, without correction for multiple comparisons.

\section{Results}

Of 1,942 HIV-positive Congolese women tested for HCV, 16 (0.83\%; 95\% CI 0.43-1.23) were HCV/HIV coinfected (Fig. 1). The majority (93.0\%) of women who were tested attended facilities in urban areas and most $(57.6 \%)$ were enrolled in a hospital setting (Table 1). Most women were under 35 years of age, with over half (52.7\%) between $25-34$ years of age, and $31.1 \%$ being 24 years or younger. The youngest participant was 15 years of age and the oldest 50 years of age. About two-thirds (67.8\%) of women included in the parent study were either divorced, separated, widowed, or never married. Very few (1.9\%) of the women in the study were smokers, and the majority $(72.4 \%)$ of women denied any alcohol consumption. The majority $(84.4 \%)$ of women had at least a secondary education. HIV RNA viral load in most (64.1\%) women was less than 1000 copies $/ \mathrm{mL}$. About half of the women involved in the study had disclosed their HIV status. The HBV prevalence in this cohort was $4.2 \%$.

Results of the bivariable and multivariable analyses are shown in Tables 2 and 3, respectively. Regarding individual characteristics, none of the HCV-positive women reported any smoking history, and the majority of women denied any alcohol consumption. In bivariable analyses, there was an association between testing positive for HBV and HCV (uOR 7.79 [2.37,25.59]). In multivariable analyses, this association with triple infection held true: the prevalence of HCV was substantially higher among women infected with hepatitis B compared to those who tested negative for HBV $(4.9 \%$ vs $0.6 \%$, aOR 13.87 [3.29,58.6]). Although not statistically significant in multivariable analyses, the prevalence of HCV was higher among women who had been on anti-retroviral 


\begin{tabular}{|c|c|c|c|}
\hline $\mathrm{HCV}+$ & $\mathbf{n} / \mathbf{N}(\%)$ & uOR $(95 \% \mathrm{CI})^{\dagger}$ & P value \\
\hline Service readiness index & & $0.95(0.90,0.99)$ & 0.019 \\
\hline \multicolumn{4}{|l|}{ Location of facility ${ }^{\S}$ attended } \\
\hline Peri-urban/rural & $2 / 135(1.5)$ & & \\
\hline Urban & $14 / 1802(0.8)$ & $0.56(0.10,2.96)$ & 0.492 \\
\hline \multicolumn{4}{|l|}{ Type of facility of care } \\
\hline Health center & $8 / 821(1.0)$ & & \\
\hline Hospital & $8 / 1116(0.7)$ & $0.72(0.25,2.07)$ & 0.544 \\
\hline \multicolumn{4}{|l|}{ PEPFAR funding } \\
\hline No & $3 / 731(0.4)$ & & \\
\hline Yes & $13 / 1206(1.1)$ & $2.62(0.69,9.88)$ & 0.156 \\
\hline \multicolumn{4}{|l|}{ Type of facility of care } \\
\hline Public & $2 / 623(0.3)$ & & \\
\hline Private for profit & $5 / 249(2.0)$ & $6.38(1.15,35.42)$ & 0.034 \\
\hline Private non-profit & 9/1065(0.8) & $2.76(0.55,13.85)$ & 0.217 \\
\hline \multicolumn{4}{|l|}{ Age } \\
\hline$\leq 24$ & $3 / 305(1.0)$ & & \\
\hline $25-34$ & 6/997(0.6) & $0.62(0.15,2.52)$ & 0.502 \\
\hline $35+$ & $7 / 589(1.2)$ & $1.22(0.31,4.82)$ & 0.778 \\
\hline \multicolumn{4}{|l|}{ Timing of testing } \\
\hline Pregnancy & $12 / 1154(1)$ & & \\
\hline Delivery & $4 / 417(1.0)$ & $0.91(0.29,2.88)$ & 0.878 \\
\hline Post-partum & $0 / 371(0)$ & - & - \\
\hline \multicolumn{4}{|l|}{ Marital status } \\
\hline Divorced/widowed/never married & $6 / 608(1.0)$ & & \\
\hline Married/cohabitating & $10 / 1283(0.8)$ & $0.79(0.28,2.20)$ & 0.646 \\
\hline \multicolumn{4}{|l|}{ Alcohol consumption } \\
\hline No & $10 / 1369(0.7)$ & & \\
\hline $1-3 /$ month & $5 / 416(1.2)$ & $1.60(0.53,4.81)$ & 0.399 \\
\hline$>2 /$ week & $1 / 106(0.9)$ & $1.20(0.14,10.33)$ & 0.869 \\
\hline \multicolumn{4}{|l|}{ Smoking } \\
\hline Previous/current & $0 / 36(0.0)$ & & \\
\hline Never & $16 / 1846(0.9)$ & - & - \\
\hline \multicolumn{4}{|l|}{ Educational level } \\
\hline Primary & $3 / 245(1.2)$ & & \\
\hline Secondary & $13 / 1348(1)$ & $0.77(0.22,2.72)$ & 0.685 \\
\hline Tertiary & $0 / 296(0.0)$ & - & - \\
\hline \multicolumn{4}{|l|}{ SES in tertiles } \\
\hline 1 (Lowest) & $4 / 607(0.7)$ & & \\
\hline 2 & $7 / 608(1.2)$ & $1.84(0.53,6.43)$ & 0.340 \\
\hline 3 (Highest) & $4 / 600(0.7)$ & $1.04(0.25,4.28)$ & 0.960 \\
\hline \multicolumn{4}{|l|}{ Primigravida } \\
\hline Yes & $1 / 195(0.5)$ & & \\
\hline No & $15 / 1700(0.9)$ & $1.82(0.22,14.65)$ & 0.576 \\
\hline \multicolumn{4}{|l|}{ Any intimate partner violence ${ }^{\#}$} \\
\hline No & $10 / 1196(0.8)$ & & \\
\hline Yes & 6/696(0.9) & $0.97(0.34,2.74)$ & 0.957 \\
\hline \multicolumn{4}{|l|}{ HIV RNA viral load } \\
\hline$\geq 1000$ copies $/ \mathrm{mL}$ & $8 / 678(1.2)$ & & \\
\hline$<1000$ copies $/ \mathrm{mL}$ & 7/1208(0.6) & $0.49(0.18,1.36)$ & 0.170 \\
\hline \multicolumn{4}{|l|}{ Duration of ART } \\
\hline$<12$ months & $11 / 846(1.3)$ & & \\
\hline 13-24 months & $1 / 215(0.5)$ & $0.34(0.04,2.74)$ & 0.311 \\
\hline$\geq 24$ months & $4 / 819(0.5)$ & $0.37(0.12,1.17)$ & 0.089 \\
\hline \multicolumn{4}{|l|}{ Disclosure of HIV status ${ }^{* *}$} \\
\hline No & 9/910(1.0) & & \\
\hline Yes & $7 / 1028(0.7)$ & $0.69(0.25,1.87)$ & 0.467 \\
\hline \multicolumn{4}{|l|}{ Continued } \\
\hline
\end{tabular}




\begin{tabular}{|l|l|l|l|}
\hline HCV + & $\mathbf{n} / \mathrm{N}(\%)$ & uOR(95\% CI $)^{\dagger}$ & P value \\
\hline HBV & $12 / 1858(0.6)$ & & \\
\hline No & $4 / 81(4.9)$ & $7.79(2.37,25.59)$ & 0.001 \\
\hline Yes & $15 / 1601(0.9)$ & & \\
\hline ART regimen & $1 / 201(0.5)$ & $0.50(0.06,4.13)$ & 0.523 \\
\hline TDF+3TC+FEV & $0 / 140(0.0)$ & - & - \\
\hline AZT +3TC+NVP & & & \\
\hline Other &
\end{tabular}

Table 2. Bivariable associations between sociodemographic, clinical and facility characteristics of the study population. *The analytical sample was derived from the enrollment data of an ongoing cluster randomized controlled trial, aimed at evaluating the effect of data-driven continuous quality improvement on long-term ART outcomes in Kinshasa, Democratic Republic of Congo. We retained participants that had available data on HCV rapid testing. SES, socio-economic status; RNA, ribonucleic acid; ART, antiretroviral therapy; HBV, hepatitis B virus; $\mathrm{uOR}$, unadjusted odds ratio; aOR, adjusted odds ratio; 95\%CI, 95\% confidence interval. ${ }^{\S}$ Facility at which participant was enrolled/tested. ${ }^{\circ}$ Calculated using principal component analysis and categorized in three groups: the lower first two quintiles, the middle quintiles, and the last two quintiles. \#Self-report of emotional or physical or sexual partner violence. ${ }^{* *}$ Self-report of disclosure of HIV status to anyone. ${ }^{\dagger} \mathrm{OR}$ and $95 \% \mathrm{CI}$ were obtained using logistic models and generalized estimating equation to adjust for potential clustering at the level of the clinic.

therapy for less than 12 months compared to those with greater than 24 months of ART (1.3\% vs. $0.5 \%$, aOR 0.49 $[0.14,1.66])$. Regarding facility characteristics, women attending private for-profit (uOR 6.38 [1.15,35.42]; aOR $19.18[2.00,183.53])$ were more likely to test positive for HCV than those attending public facilities. Additionally, women attending facilities in urban areas were less likely to test positive for HCV as opposed to those attending facilities in peri-urban/rural areas (aOR $0.35[0.07,1.84])$. Women who attended facilities with PEPFAR funding (aOR $6.72[1.35,33.42]$ ) or facilities with lower capacity to provide general maternal and child health services were also more likely to test positive for HCV. Each percent point increase in the SRI was associated with an $8 \%$ lower odds in HCV prevalence (aOR:0.92 [0.86,0.98]), relatively.

\section{Discussion}

The prevalence of HCV/HIV co-infection among pregnant and postpartum women living in Kinshasa Province in the DRC was $0.83 \%$. This is consistent with prior estimates of HCV infection in the DRC, irrespective of HIV status ${ }^{2}$. This study also found that the odds of HCV infection was 13.87 times higher in HBV-infected women than HBV-uninfected women, raising concerns for a vulnerable population of $\mathrm{HCV} / \mathrm{HBV} / \mathrm{HIV}$ triple-infected women.

Factors contributing to triple-infection are not entirely clear and studies investigating outcomes in those with triple infection of $\mathrm{HCV} / \mathrm{HBV} / \mathrm{HIV}$ are limited. A recent study in Nigeria found that in those with HCV/ $\mathrm{HBV} / \mathrm{HIV}$ triple-infection, CD4+ counts are significantly lower as compared to those with HIV infection alone. Individuals with triple-infection also achieve less benefit with anti-retroviral therapy than those with monoHIV infection ${ }^{15}$.

Effective treatments for HCV are available but are not easily accessible for most inhabitants of the DRC. While MTCT of HCV is relatively low (5-7\%) in HCV mono-infected mothers, rates of MTCT of HCV are higher (10-20\%) among HCV/HIV co-infected women ${ }^{7}$. However, HCV treatment is not recommended during pregnancy and effective interventions to prevent MTCT in HCV-infected pregnant women have yet to be identified. HCV-infected infants are subsequently at high risk for liver disease progression ${ }^{16}$. It is therefore of utmost importance that future efforts focus on the treatment of HCV-infected women of childbearing age prior to pregnancy in African settings. In addition, measures for PMTCT of HIV and HBV should be prioritized in cases of $\mathrm{HCV} / \mathrm{HBV} / \mathrm{HIV}$ triple infection to reduce the chances of these infections in women and their infants.

This study highlights facility-level, socio-demographic and clinical factors associated HCV/HIV co-infection in pregnant and breastfeeding women. HCV/HIV co-infected women were more likely to present for care during pregnancy than in the postpartum period, which argues that pregnant women should be targeted for screening during a time when they are more likely to seek care. HCV/HIV co-infected women were also younger and more likely to live in urban areas. We found that women who attended private facilities in general, and for-profit facilities in particular, were more likely to be HCV-positive. The primary route of HCV transmission in African settings is via nosocomial spread, and thus facility characteristics (sterilization of equipment, medical procedures performed, etc.) are important to examine in depth in future studies ${ }^{17}$. As expected, women attending facilities with a higher capacity to provide general maternal and child health services (high SRI score) were less likely to be infected with HCV. We also found that HCV-positive women were more likely to attend facilities with direct PEPFAR funding. PEPFAR supported facilities are typically large urban hospitals and generally provide better HIV care services ${ }^{13}$, thus it is possible that this association is due to residual confounding.

The strength of this study lies in its contribution to a field with a current paucity in data. There is very little research available investigating $\mathrm{HIV} / \mathrm{HCV}$ co-infection in pregnant Congolese women, making this study one 


\begin{tabular}{|c|c|c|c|c|c|c|}
\hline & $\begin{array}{l}\text { aOR }(95 \% \mathrm{CI})^{\dagger} \\
\text { Individual } \\
\text { characteristics }\end{array}$ & P value & $\begin{array}{l}\text { aOR }(95 \% \mathrm{CI}) \\
\text { Facility characteristics }\end{array}$ & P value & $\begin{array}{l}\text { aOR }(95 \% \mathrm{CI}) \\
\text { Combined } \\
\text { characteristics }\end{array}$ & P value \\
\hline Service readiness index & $0.94(0.89,0.99)$ & 0.017 & $0.94(0.89,0.99)$ & 0.021 & $0.92(0.86,0.98)$ & 0.010 \\
\hline \multicolumn{7}{|c|}{ Location of facility attended } \\
\hline \multicolumn{7}{|l|}{ Peri-urban/rural } \\
\hline Urban & & & $0.47(0.11,2.02)$ & 0.309 & $0.35(0.07,1.84)$ & 0.216 \\
\hline \multicolumn{7}{|l|}{ Type of facility of care } \\
\hline \multicolumn{7}{|l|}{ Health center } \\
\hline Hospital & & & $1.17(0.39,3.50)$ & 0.782 & $1.05(0.28,3.93)$ & 0.944 \\
\hline \multicolumn{7}{|l|}{ PEPFAR funding } \\
\hline \multicolumn{7}{|l|}{ No } \\
\hline Yes & & & $2.93(0.89,9.69)$ & 0.078 & $6.72(1.35,33.42)$ & 0.020 \\
\hline \multicolumn{7}{|l|}{ Type of facility of care } \\
\hline \multicolumn{7}{|l|}{ Public } \\
\hline Private for profit & & & $6.40(1.35,30.22)$ & 0.019 & $19.18(2.00,183.53)$ & 0.010 \\
\hline Private non-profit & & & $2.65(0.64,11.03)$ & 0.180 & $7.15(0.83,61.68)$ & 0.074 \\
\hline \multicolumn{7}{|l|}{ Age } \\
\hline \multicolumn{7}{|l|}{$\leq 24$} \\
\hline $25-34$ & $0.49(0.10,2.33)$ & 0.369 & & & $0.50(0.10,2.55)$ & 0.404 \\
\hline $35+$ & $1.41(0.31,6.48)$ & 0.656 & & & $1.51(0.30,7.54)$ & 0.612 \\
\hline \multicolumn{7}{|l|}{ Timing of testing } \\
\hline \multicolumn{7}{|l|}{ Pregnancy } \\
\hline \begin{tabular}{|l|} 
Delivery \\
\end{tabular} & $0.74(0.20,2.75)$ & 0.652 & & & $0.62(0.16,2.39)$ & 0.49 \\
\hline Post-partum & - & - & & & - & - \\
\hline \multicolumn{7}{|l|}{ SES in tertile } \\
\hline \multicolumn{7}{|l|}{ 1(Lowest) } \\
\hline 2 & $1.71(0.47,6.18)$ & 0.415 & & & $1.74(0.45,6.68)$ & 0.419 \\
\hline 3 (Highest) & $1.53(0.35,6.78)$ & 0.573 & & & $1.90(0.41,8.91)$ & 0.416 \\
\hline \multicolumn{7}{|l|}{ Primigravida } \\
\hline \multicolumn{7}{|l|}{ Yes } \\
\hline No & $2.16(0.23,20.21)$ & 0.498 & & & $1.95(0.20,19.25)$ & 0.566 \\
\hline \multicolumn{7}{|l|}{ Duration of ART } \\
\hline \multicolumn{7}{|l|}{$<12$ months } \\
\hline 13-24 months & - & - & & & - & - \\
\hline$\geq 24$ months & $0.44(0.13,1.46)$ & 0.180 & & & $0.49(0.14,1.66)$ & 0.249 \\
\hline \multicolumn{7}{|l|}{ HBV } \\
\hline \multicolumn{7}{|l|}{ No } \\
\hline Yes & $12.07(3.31,44.05)$ & $<0.001$ & & & $13.87(3.29,58.6)$ & 0.001 \\
\hline
\end{tabular}

Table 3. Multivariable associations between sociodemographic, clinical and facility characteristics of the study population. The analytical sample was derived from the enrollment data of an ongoing cluster randomized controlled trial, aimed at evaluating the effect of data-driven continuous quality improvement on long-term ART outcomes in Kinshasa, Democratic Republic of Congo. We retained participants that had available data on HCV rapid testing. SES, socio-economic status; RNA, ribonucleic acid; ART, antiretroviral therapy; HBV, hepatitis B virus; $\mathrm{uOR}$, unadjusted odds ratio; aOR, adjusted odds ratio; 95\% CI, 95\% confidence interval. ${ }^{\S}$ Facility at which participant was enrolled/tested. ${ }^{~ C a l c u l a t e d ~ u s i n g ~ p r i n c i p a l ~ c o m p o n e n t ~ a n a l y s i s ~ a n d ~}$ categorized in three groups: the lower first two quintiles, the middle quintiles, and the last two quintiles. ${ }^{\dagger} \mathrm{OR}$ and $95 \% \mathrm{CI}$ were obtained using logistic models and generalized estimating equation to adjust for potential clustering at the level of clinic.

of the first to evaluate this specific population. This study also considers both typical HCV risk factors as well as factors specific to the facilities in which women seek care.

A primary limitation of this study was its generalizability, as data was obtained from pregnant women only within Kinshasa Province and therefore may not generalizable outside of this region. Additionally, as with any cross-sectional study, we were not able to imply causation. This study was also limited by a lack of confirmatory HCV RNA testing, with only HCV antibody status testing evaluated. Thus, we were not able to decipher timing of infection (active vs. past). An additional limitation of this study was the testing method used to evaluate HCV status in the patient population. Although the Abbott Determine HCV test has been verified in other 
settings (in Nigeria, Georgia, Cambodia, and Belgium as referenced), no data is available regarding its analytical performance in the DRC.

\section{Conclusion}

The prevalence of HCV/HIV co-infection among pregnant Congolese women in this study was consistent with previous estimates of HCV prevalence in the region. HCV/HIV co-infected women tended to be younger, live in urban areas, and seek care in private and PEPFAR-funded clinics. Facility-related risk factors should be further researched, as nosocomial transmission is a major risk factor for HCV infection in African settings. HCV/HIV co-infected women had a much higher odds of being triple-infected with HBV as well, a phenomenon that warrants further investigation. Future studies should target women during pregnancy for HCV screening in order to implement curative treatment post-pregnancy and thus prevent transmission in subsequent pregnancies.

Received: 24 April 2020; Accepted: 5 January 2022

Published online: 26 January 2022

\section{References}

1. Mora, N. et al. A synthesis of hepatitis C prevalence estimates in Sub-Saharan Africa: 2000-2013. BMC Infect. Dis. 16, 283 (2016).

2. Parr, J. B. et al. An efficient, large-scale survey of hepatitis $\mathrm{C}$ viremia in the democratic republic of the congo using dried blood spots. Clin. Infect. Dis. 66, 254-260 (2018).

3. Sulkowski, M. S. et al. Rapid fibrosis progression among HIV/hepatitis C virus-co-infected adults (2007).

4. Collins, S. et al. Liver-related deaths in persons infected with the human immunodeficiency virus: The D:A: D study. Arch. Intern. Med. 166, 1632-1641 (2006).

5. Mohd Hanafiah, K., Groeger, J., Flaxman, A. D. \& Wiersma, S. T. Global epidemiology of hepatitis C virus infection. Hepatology 57, 1333-1342 (2013).

6. Azevedo, T. C. L., Zwahlen, M., Rauch, A., Egger, M. \& Wandeler, G. Hepatitis C in HIV-infected individuals: A systematic review and meta-analysis of estimated prevalence in Africa. J. Int. AIDS Soc. 19, 20711 (2016).

7. Yeung, L. Mother-to-infant transmission of hepatitis C virus. Hepatology 34, 223-229 (2001).

8. Mpody, C. et al. Hepatitis B infection among pregnant and post-partum women living with HIV and on antiretroviral therapy in Kinshasa, DR Congo: A cross-sectional study. PLoS ONE 14, e0216293 (2019).

9. Yotebieng, M. et al. Continuous quality improvement interventions to improve long-term outcomes of antiretroviral therapy in women who initiated therapy during pregnancy or breastfeeding in the Democratic Republic of Congo: Design of an open-label, parallel, group randomized trial. BMC Health Serv. Res. 17, 1-7 (2017).

10. Hockstein, E. Health Facility Assessment of Service Availability and Readiness Service Availability and Readiness Assessment (SARA) An annual monitoring system for service delivery Reference Manual. www.who.int (2015).

11. Vetter, B. N. et al. Sensitivity and specificity of rapid diagnostic tests for Hepatitis C virus with or without HIV coinfection: A multicentre laboratory evaluation study. J. Infect. Dis. https://doi.org/10.1093/infdis/jiaa389 (2020).

12. SD BIOLINE HCV|Abbott Point of Care Testing. https://www.globalpointofcare.abbott/en/product-details/sd-bioline-hcv.html.

13. Yotebieng, M. et al. HIV viral suppression among pregnant and breastfeeding women in routine care in the Kinshasa province: A baseline evaluation of participants in CQI-PMTCT study. J. Int. AIDS Soc. 22, e25376 (2019).

14. Alere Determine HBsAg|Abbott Point of Care Testing. https://www.globalpointofcare.abbott/en/product-details/determine-hbsag. html.

15. Ogwu-Richard, S. O., Ojo, D. A., Akingbade, O. A. \& Okonko, I. O. Triple positivity of HBsAg, anti-HCV antibody, and HIV and their influence on CD4+ lymphocyte levels in the highly HIV infected population of Abeokuta, Nigeria. Afr. Health Sci. 15, 719-727 (2015).

16. Mack, C. L. et al. NASPGHAN practice guidelines: Diagnosis and management of Hepatitis C infection in infants, children, and adolescents. J. Pediatric Gastroenterol. Nutr. https://doi.org/10.1097/MPG.0b013e318258328d (2012).

17. Lemoine, M., Eholié, S. \& Lacombe, K. Reducing the neglected burden of viral hepatitis in Africa: Strategies for a global approach. J. Hepatol. 62, 469 (2015).

\section{Acknowledgements}

This research was supported by the President's Emergency Plan for AIDS Relief (PEPFAR) and the National Institute of Health and Child Development (NIHCD 1R01H87993). MY and CM are partially supported by the National Institutes of Health (NIAID U01AI096299). PT received salary support from the National Institutes of Health (NIAID K08AI148607) and from an ASTMH/Burroughs-Wellcome Fellowship in Tropical Medicine, outside of this work. The funders had no role in study design, data collection, data collection, data analysis and interpretation, preparation of the manuscript, or decision to submit. We thank all of the pregnant and breastfeeding women who contributed to the findings of this study. We acknowledge the contribution of the following site investigators of the CQI-PMTCT study team: Godelive Aitikalema, Ali Alisho, Elysée Bayayana, Fabrice Bumwana, Pierre Dianzenza, Jean Claude Dinanga, Georges Kihuma, Willy Lukumu, Fidèle Lumande, Zouzou Masevo, Fanny Matadi, Rachel Mushiya, Marie Therèse Mwela, José Nlandu, Pearl Tenatena and Marie Tshibuabua. We are grateful to participating clinics, provincial and national health authorities. We also acknowledge the support we have received from the administrative staff of the Ohio State University and Kinshasa School of Public Health.

\section{Author contributions}

M.Y., F.B., and E.O. conceptualized the study and acquired funding. C.M., M.T., N.L.R.R., F.M., B.K., E.O., and M.Y. acquired the data. C.M. and M.Y. performed the data analysis. C.M., P.T., W.S. and M.Y. drafted the first manuscript. All authors read and approved the final manuscript.

\section{Competing interests}

The authors declare no competing interests. 


\section{Additional information}

Supplementary Information The online version contains supplementary material available at https://doi.org/ 10.1038/s41598-022-05014-3.

Correspondence and requests for materials should be addressed to P.T.

Reprints and permissions information is available at www.nature.com/reprints.

Publisher's note Springer Nature remains neutral with regard to jurisdictional claims in published maps and institutional affiliations.

(c) (1) Open Access This article is licensed under a Creative Commons Attribution 4.0 International License, which permits use, sharing, adaptation, distribution and reproduction in any medium or format, as long as you give appropriate credit to the original author(s) and the source, provide a link to the Creative Commons licence, and indicate if changes were made. The images or other third party material in this article are included in the article's Creative Commons licence, unless indicated otherwise in a credit line to the material. If material is not included in the article's Creative Commons licence and your intended use is not permitted by statutory regulation or exceeds the permitted use, you will need to obtain permission directly from the copyright holder. To view a copy of this licence, visit http://creativecommons.org/licenses/by/4.0/.

(C) The Author(s) 2022

\section{CQI-PMTCT study team}

\section{Godelive Aitikalema ${ }^{5}$, Ali Alisho ${ }^{5}$, Elysée Bayayana ${ }^{5}$, Fabrice Bumwana ${ }^{5}$, Pierre Dianzenza ${ }^{5}$, Jean Claude Dinanga ${ }^{5}$, Georges Kihuma ${ }^{5}$, Willy Lukumu ${ }^{5}$, Fidèle Lumande ${ }^{5}$, Zouzou Masevo $0^{5}$, Fanny Matadi ${ }^{5}$, Rachel Mushiya ${ }^{5}$, Marie Therèse Mwela ${ }^{5}$, José Nlandu ${ }^{5}$, Pearl Tenatena ${ }^{5}$ \& Marie Tshibuabua ${ }^{5}$}

